The most likely role of HLA in determining susceptibility to disease is through immune response genes that determine recognition of a particular antigenic determinant. Evidence for this concept is based mostly on the close resemblance of HLA to mouse $\mathrm{H}-2$, which is known to control immune responses.

Among the models available for the study of spontaneously appearing autoimmunity in animals, the New Zealand (NZ) strains of mice have been by far the most profitable. The NZB strain spontaneously develops haemolytic anaemia, and the hybrid obtained by crossing NZB with the apparently normal NZW strain results in an autoimmune disease closely resembling human systemic lupus erythematosus (SLE). Extensive efforts have been made to sort out the several genetic factors controlling these autoimmune disorders, but a clear definition has not yet been obtained. To complicate matters, NZ mice contain abundant type $C$ virus particles. Viral genes code for glycoprotein which closely resembles a mouse glycoprotein, and the possibility was raised that antibody originally induced to viral proteins may cross react with the comparable mouse antigen. However, S. Datta (Tufts University) showed that viral genes segregate independently of 'autoimmunity' genes, removing the possibility of a cause-andeffect relationship between viral infection and autoimmunity.

A more likely possibility to explain the spontaneous development of autoimmune disease in NZ mice is a fundamental disorder in immunological regulation. H. Cantor (Harvard Medical School) demonstrated an early deficiency in the subset of $\mathrm{T}$ lymphocytes responsible for immunological equilibrium. These cells, recognised by their antigenic surface markers, Ly $123^{+}$, produce feedback suppression of antibody production. N. Talal (University of California, San Francisco) further showed that the paucity of suppressor $\mathrm{T}$ cells in $\mathrm{NZ}$ mice can be at least partially remedied by thymic extracts. Another view of the fundamental NZ abnormality emerged from experiments of $\mathbf{H}$. Morse and T. Chused (National Institutes of Health). B cells of NZB mice show spontaneous polyclonal activation even in the absence of $T$ cells. These findings suggest that $\mathrm{NZ}$ mice suffer from an intrinsic lesion in their $\mathbf{B}$ cells. There may be a more basic stem cell defect affecting both regulatory $T$ cells and $B$ cells.

In contrast to the multiple autoimmunities present in $\mathrm{NZ}$ mice, a single autoimmune disorder can be demonstrated in the OS strain of chickens. These animals spontaneously develop a classical form of chronic thyroiditis. L. Bacon (Wayne State University)

\title{
DNA structure and transcription
}

\section{from Andrew Travers}

THE mechanisms controlling transcription during the development of different bacteriophages are extremely diverse. Yet another solution has emerged from the studies of Rothman-Denes and her colleagues on coliphage N4. N4, in common with Bacillus subtilis phage PBS2, but unlike all other commonly studied phages, does not apparently require the host RNA polymerase for any viral transcription. Instead the virus contains a virus-coded transcriptase of novel structure. This enzyme consists of a single protein chain about 4,000 amino acids long and is thus one of the largest polypeptides so far identified.

How does this enzyme function? Its equally novel template requirements are now reported in the latest paper from Chicago (Falco et al. Proc. natn. Acad. Sci. U.S.A. 75, 3220 ; 1978). In vitro N4 RNA polymerase strongly prefers N4 DNA, which it copies asymmetrically, producing transcripts corresponding to those RNA molecules synthesised in vivo immediately after phage infection. However, in contrast to other bacteriophages, the enzyme has the virtually absolute requirement that this template be denatured. Another pointer to the unusual nature of the specificity of the enzyme is the observation that in vivo N4 RNA synthesis is specifically inhibited by coumermycin, a drug whose target is DNA gyrase, the enzyme responsible for inserting negative superhelical turns into closed circular duplex DNA. These results suggest that DNA gyrase must modify the structure of N4 DNA before it can be transcribed by the virion RNA polymerase. Consequently, the authors propose that superhelical DNA may be the required natural template for the enzyme.

Assuming this hypothesis to be correct, we have to ask how the introduction of negative twists into presumably closed circular N4 DNA

Andrew Travers is at the MRC Laboratory for Molecular Biology, Cambridge.

found that the trait is polygenic but is significantly linked to the B locus (the chicken's MHC) in certain families. In other families the B locus linkage may be obscured due to over-riding effects of other genes. Other genetic defects have been found in the OS thymus and in the thyroid gland itself. so dramatically promotes transcripttion. The best current analogy comes from studies on Escherichia coli RNA polymerase. Before initiating transcription this enzyme recognises a promoter site extending over a region of at least 40-50 base pairs, and subsequently effects a change in DNA conformation at the binding site, a process termed promoter opening. It is well established that increasing the superhelical density of phage DNA templates substantially enhances the rate of transcription initiation by the bacterial polymerase. This enhancement could result from a direct facilitation of enzyme-mediated promoter opening. Alternatively the introduction of superhelical turns could alter the parameters which the enzyme recognises in the DNA template sufficiently to change the initial affinity of the polymerase for a promoter site.

At present the available experimental evidence does not distinguish between these possibilities. However, Levitt (Proc natn. Acad, Sci. U.S.A. 75,$640 ; 1978$ ) has recently calculated that supercoiling can, in theory, alter the number of base pairs per turn of the double helix by as much as $5 \%$. If this result is applicable to the real world, it is difficult to escape the implication that the spatial arrangement of the DNA sequence in a supercoiled molecule differs substantially from that in a relaxed one. Consequently unless one invokes the additional ad hoc assumption that RNA polymerase possesses a similar molecular flexibility it seems highly plausible that the detail of the initial contacts of the enzyme with a given promoter site will strongly depend on the extent of superhelicity of the template. However, the capacity of the bacterial polymerase for differential promoter recognition can be extensively modulated by factors other than subtle variations in DNA structure. Under these circumstances the N4 virion RNA polymerase promises to provide an attractive simpler system to test for the effects of supercoiling on promoter recognition and opening.

On the basis of the many studies of spontaneously developing autoimmune disease in animals, three types of genetic eccentricity can be recognised. One is an increase in immunological responsiveness, possibly due to the presence of immine response genes within the MHC. Such a 